\title{
Análise de Rede Brasileira de Comunicação Óptica Integrada com Rede de Satélites LEO
}

\author{
Helvécio M. Almeida, Eduardo M. G. de Queiroz e Amílcar C. César
}

\begin{abstract}
Resumo - A simulação do desempenho uma rede óptica WDM integrada com uma rede de satélites LEO (low earth orbit) cobrindo o território brasileiro é descrita neste artigo. A rede óptica é formada por 44 nós e 56 enlaces e a de satélites por 14 estações terrestres, das quais 5 possuem interconexão com a rede óptica, cobrindo o território por meio de 3 trajetórias orbitais. Há enlaces entre satélites e estações terrestres e entre satélites. Fatores de degradação da qualidade do sinal são considerados nas duas redes, como chuva, PMD e ruído ASE. O desempenho da rede integrada é avaliado por meio de probabilidade de bloqueio de conexões e throughput.
\end{abstract}

Palavras-chave - Rede óptica WDM, rede de satélites LEO, alocação de recursos, fator de degradação de sinal, algoritmo de otimização.

\begin{abstract}
The simulation of an optical WDM network integrated with a LEO satellite network covering the Brazilian territory is described in this article. The optical network is composed of 44 nodes and 56 links and the satellite network of 14 terrestrial stations, which 5 have interconnection with the optical network, covering the territory by 3 orbital paths. There are links between satellites and terrestrial stations and intersatellites. Degradation factors of signal quality are considered in the networks, such as rain, PMD and ASE. The performance of the integrated network is evaluated by means of blocking probability of connection request and throughput.
\end{abstract}

Keywords-WDM optical network, LEO satellites network, resource allocation, physical impairments, optimization.

\section{INTRODUÇÃO}

A interligação de redes de telecomunicações, motivada por tráfego intenso, começa a exigir gerência integrada para oferecer serviços com qualidade e reduzir custos. A interligação de redes óptica e via satélite se destaca pela capacidade de transportar grandes volumes de tráfego e pela capacidade de integrar regiões de acesso difícil.

As redes ópticas operam com base na multiplexação por divisão de comprimentos de onda (WDM- wavelength division multiplexing, que agrega vários comprimentos de onda em uma única fibra.

Por outro lado, as constelações de satélites operadas por vários consórcios utilizam órbitas baixa (LEO_ low earth

Helvécio M. Almeida, Eduardo M. G. de Queiroz e Amílcar C. César, Universidade de São Paulo, Escola de Engenharia de São Carlos, Depto. Eng. Elétrica, São Carlos, SP. E-mails: asah@terra.com.br, \{equeiroz;amilcar\}@ sel.eesc.usp.br.

Esta pesquisa foi parcialmente financiada pela Capes, CNPq e FAPESP (Projeto Tidia-KyaTera, Lab. LightWays, proc. 03/08269-7) orbit), com altitudes entre 500 e $1500 \mathrm{~km}$ [1], média, com altitudes entre 8.000 e $18.000 \mathrm{~km}$ e geoestacionária, com altitude média de $35.000 \mathrm{~km}$ [2]. O uso de satélites para comunicação é particularmente útil no Brasil por causa da dimensão territorial e da distribuição heterogênea da população.

Geralmente, os dois tipos de redes são administrados por operadoras distintas, que alocam os recursos de acordo com disponibilidade e características. Por conta da demanda heterogênea de largura de faixa, o tráfego servido pela interconexão das duas redes pode ser forçado a trafegar por enlaces de capacidades distintas, podendo haver bloqueio. Neste caso, o estabelecimento de rotas e alocação de recursos deve ser otimizado para garantir atendimento da demanda.

O problema de alocação de rota e recursos, como comprimento de onda, (RWA - routing and wavelength assignment) em redes WDM tem sido extensivamente abordado [3]-[5]. Em redes de satélites, técnicas de otimização dos recursos também têm despertado interesse em face da complexidade e custo [1], [6].

O sinal que propaga pelas duas redes sofre degradação por causa das características físicas do meio de propagação e dos diversos dispositivos [4], [5]. São exemplos de causa de degradação do sinal a chuva e o ruído ASE (amplified stimulated emission) em amplificadores ópticos. O efeito da degradação sobre o desempenho do sistema pode ser avaliado pelo aumento da taxa de erro de bit (BER- bit error ratio).

Em artigo anterior [7], a interligação entre os dois tipos de redes foi investigada, porém sem a inclusão de fatores de degradação de sinal (restrições físicas). Naquele artigo, foi considerado somente satélite geoestacionário.

A contribuição deste artigo é a interligação das redes óptica e de satélites LEO considerando fatores físicos de degradação de qualidade do sinal (restrições físicas). Nos enlaces via satélites são considerados os efeitos causados por chuva e nos ópticos, efeitos como ASE, PMD (polarization mode dispersion) e ruído crosstalk da chave são incluídos no cálculo da BER. As simulações de desempenho sob vários cenários são feitas em redes cobrindo o território nacional. As regiões norte e centro-oeste são atendidas mais por satélites do que por fibra óptica.

\section{DESCRIÇÃO DA REDE INTEGRADA}

A disposição geográfica dos nós que compõem a rede integrada é a mesma adotada em [7], conforme mostra a Figura 1. As características de cada rede são descritas a 
seguir.

\section{A. Rede Óptica}

A rede óptica é formada por 44 nós e 56 enlaces. $\mathrm{O}$ acesso (interconexão) à rede de satélites é realizado por meio de cinco nós. Em cada enlace há oito comprimentos de onda com largura de faixa de 2,5 Gbps cada um. Os amplificadores ópticos estão situados a cada $70 \mathrm{~km}$. O algoritmo Dijskstra é utilizado para encontrar o menor caminho entre os nós origem e destino e o first-fit para escolher o comprimento de onda disponível para estabelecer a conexão. Cada solicitação de conexão é enquadrada em seis tipos de serviço, de acordo com a largura de faixa, conforme mostra a Tabela I. Para efeito prático, quanto maior for a largura de faixa solicitada, menor será a probabilidade de geração.

TABEla I. SERVIÇOS, LARGURAS DE FAIXA E PROBABILIDADE DE GERAÇÃO.

\begin{tabular}{|c|c|c|}
\hline $\begin{array}{c}\text { Classe de } \\
\text { Serviço }\end{array}$ & $\begin{array}{c}\text { Largura de Faixa } \\
\text { (Mbps) }\end{array}$ & $\begin{array}{c}\text { Probabilidade de } \\
\text { Geração (\%) }\end{array}$ \\
\hline I & 155,52 & 59 \\
\hline II & 622,08 & 15 \\
\hline III & 933,12 & 10 \\
\hline IV & 1.244 & 8 \\
\hline V & 1.866 & 5 \\
\hline VI & 2.488 & 3 \\
\hline
\end{tabular}

\section{B. Arquitetura dos Nós}

A arquitetura dos nós 1, 5, 7, 9, 11 a 15, 17, 18, 20, 21, 23 a 25, 28 a 34, 38, 41, 43 e 44 é a do tipo 2, adotada em [3]. É formada por OXC (optical cross-connect), não dispõem de conversores de comprimento de onda e são capazes de agregar, entre os mesmos, no máximo 16 diferentes conexões em um comprimento de onda (traffic grooming). A agregação é feita em camadas eletrônicas da rede e, portanto, estes nós realizam conversão óptica-eletrônica-óptica. Estes nós estão localizados em regiões onde o número de habitantes servidos ultrapassa 1 milhão. Os nós que possuem apenas conexão com satélites são capazes de gerar apenas solicitações de 155 Mbps, que é a largura de faixa máxima suportada pelos canais dos satélites.

\section{Rede de Satélites LEO}

A rede de satélites é do tipo LEO com altitude orbital 1500 $\mathrm{km}$. Cada um dos seis satélites dispõe de quatro transceptores, cuja taxa máxima é 155 Mbps cada. Há 14 estações terrestres, das quais 5 são interligadas à rede óptica. A movimentação em órbita forma uma malha em que as distâncias entre os satélites são fixas. Para que qualquer região do território esteja sempre coberta, a malha é formada por 6 satélites, que podem formar enlace entre eles (ISL-inter-satellite link). A Figura 1 mostra também as estações terrestres correspondentes a cada órbita. Por exemplo, os satélites da órbita 1 realizam conexão apenas com as estações terrestres representadas pelos nós 48 a 53. O algoritmo Dijskstra considera a rede de forma global, incluindo os satélites como nós.

Três tipos de handovers ocorrem em redes de satélite LEO: o handover entre satélites (intersatellite), que é a mudança de satélites para uma determinada conexão; o handover de enlace (link), quando há a necessidade de um novo roteamento de conexão na rede; spotbeam handover, que considera a mudança de uma conexão entre spotbeams de um mesmo satélite [2].

Neste trabalho, devido à configuração da interligação da rede óptica com a rede de satélite, não há bloqueio de conexão devido ao handover porque são consideradas apenas as conexões oriundas das estações terrestres da rede brasileira. As estações terrestres são fixas e não solicitam recursos por passagem de estações móveis de uma área coberta por um satélite para outra. Desta maneira, as conexões atendidas por um satélite serão atendidas por outro satélite no momento de mudança da área de cobertura devido à movimentação orbital. Consideramos que, no momento desta mudança, todas as conexões atendidas na área de cobertura anterior são automaticamente atendidas pelo próximo satélite e que não há mudança de rotas entre os satélites (ISL) das conexões alocadas, já que são computadas por meio do algoritmo Dijskstra.

A Figura 2 mostra as estações terrestres fixas e a movimentação orbital dos satélites. Neste exemplo, a conexão 1 é atendida pelo satélite 1 e a conexão 2 é atendida pelo satélite 3. Quando há movimentação do satélite 3, a conexão 2 passa então a ser atendida pelo satélite 2 . Consideramos que o satélite 2 atenderá as mesmas conexões que o satélite 3 já atende e que os recursos da rede de 6 satélites são solicitados apenas pela demanda da rede óptica.

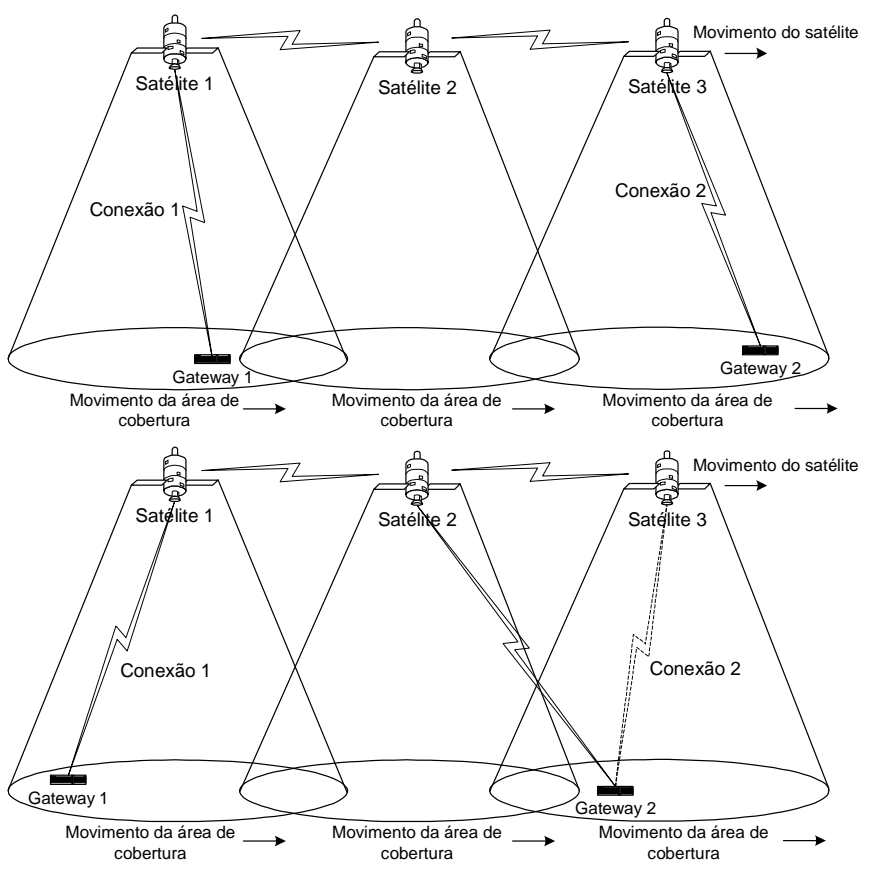

Figura 2. Exemplo de conexões entre satélites e nós terrestres.

\section{MODELO DE RESTRIÇÃO FÍSICA DA REDE ÓPTICA}

O modelo da rede óptica inclui vários fatores de degradação da qualidade do sinal, cujos valores são listados na Tabela II da Seção V. Os efeitos correspondentes são incluídos no cálculo da relação sinal-ruído óptica (OSNR), a partir da qual a BER dos enlaces é calculada por 


$$
B E R=0,5 \operatorname{ercf}\left(\frac{Q}{\sqrt{2}}\right)
$$

na qual

$$
Q=\sqrt{\frac{B_{o}}{B_{e}}} \frac{2 O S N R}{\sqrt{4 O S N R+1}+1},
$$

$B_{o}$ é a largura de banda óptica e $B_{e}$ é a largura de banda elétrica. A OSNR é calculada de acordo com o modelo desenvolvido em [8]. Por falta de espaço as expressões de [8] não são reproduzidas aqui.

Para os nós que agregam tráfego, descritos na Seção II.B, o valor da BER é zerado e reiniciado no enlace seguinte. Se uma conexão dispõe de enlace com satélite, a BER é contabilizada até a estação terrestre que está interligada com o satélite.

\section{Modelo de AtenuaÇão POR ChUVA}

Nos enlaces de satélites o modelo de restrições físicas é baseado na atenuação por chuva via cálculo da relação sinalruído $\mathrm{C} / \mathrm{N}$ (carrier-to-noise ratio). As relações $\mathrm{C} / \mathrm{N}$ para uplink e downlink são calculadas com o modelo apresentado em [2]:

$\left(\frac{C}{N}\right)_{u}=10 \log \left(G_{T} P_{T}\right)-20 \log \left(\frac{4 \pi R_{u}}{\lambda_{u}}\right)+10 \log \left(\frac{G_{S}}{T_{S}}\right)-10 \log \left(K B_{S}\right)+10 \log \left(L_{u}\right)$

$\left(\frac{C}{N}\right)_{d}=10 \log \left(G_{S} P_{S}\right)-20 \log \left(\frac{4 \pi R_{d}}{\lambda_{d}}\right)+10 \log \left(\frac{G_{d}}{T_{d}}\right)-10 \log \left(K B_{r}\right)+10 \log \left(L_{d}\right)$

nas quais os índices $u$ e $d$ se referem a uplink e downlink; $G_{T} \mathrm{e}$ $P_{T}$ são o ganho e a potência da estação terrestre; $R_{u}$ e $R_{d}$ são as distâncias entre o satélite e a estação terrestre; $\lambda_{u}$ e $\lambda_{d}$ são comprimentos de onda dos sinais; $G_{s}$ e $T_{s}$ são o ganho e a temperatura de ruído do satélite; $G_{d}$ e $T_{d}$ são o ganho e a temperatura de ruído do receptor terrestre; $L_{d}$ e $L_{u}$ são os fatores de atenuação por chuva; $B_{s}$ é a largura de banda do transponder do satélite e $B_{r}$ é a largura de banda do receptor terrestre; $K=1,38.10^{-23}$ é a constante de Boltzmann.

A atenuação por $C / N$ provocado por chuva para os links de satélites é modelada com o modelo apresentado por [2]. As expressões de [2] não são reproduzidas aqui por falta de espaço. A atenuação causada por chuva calculada por [2] é incluída em (3) e (4) por $L_{u}$, no uplink e $L_{d}$, no downlink.

Vários fatores de degradação da qualidade do sinal nas duas redes podem ser incluídos no algoritmo. Por falta de espaço deixamos de analisar o impacto de alguns deles, como o Doppler na rede via satélites, sobre o desempenho da rede integrada.

\section{PARÂMETROS UTILIZADOS}

\section{1) Parâmetros da rede óptica}

Os parâmetros de simulação utilizados na rede óptica estão na Tabela II.

\begin{tabular}{|c|c|}
\hline Taxa de bit de cada canal & $10 \mathrm{Gbps}$ \\
\hline Largura de Banda Óptica $\left(B_{o}\right)$ & $70 \mathrm{GHz}$ \\
\hline Potência de sinal por canal & $1 \mathrm{~mW}$ \\
\hline Valor do ruído crosstalk da chave $\left(X_{s w}\right)$ & $-25 d B$ \\
\hline $\begin{array}{l}\text { Perda do } \\
\text { multiplexador/demultiplexador }\left(L_{m} / L_{d m}\right)\end{array}$ & $-4 d B$ \\
\hline Perda na chave $\left(L_{s w}\right)$ & $-8 d B$ \\
\hline Perda no conector $\left(L_{\text {tap }}\right)$ & $-1 d B$ \\
\hline Ganho do EDFA $\left(G_{\text {in }}, G_{\text {out }}\right)$ & $12 d B, 6 d B$ \\
\hline Fator ASE do EDFA $\left(n_{s p}\right)$ & 1,2 \\
\hline Parâmetro PMD da fibra $\left(D_{P M D(k)}\right)$ & $0,5 \mathrm{ps} /(\mathrm{km})^{1 / 2}$ \\
\hline Ganho desejado do DRA & $20 d B$ \\
\hline $\begin{array}{l}\text { Comprimentos de onda de bombeio (em } \\
\mathrm{nm} \text { ) }\end{array}$ & 1410,1450 e 1500 \\
\hline Potências de bombeio (em W) & $0,607,0,209$ e 0,01 \\
\hline Limiar Tolerável da OSNR $(B E R)$ & $7,4 d B\left(\right.$ para uma BER $\left.10^{-9}\right)$ \\
\hline Fator de polarização $\left(K_{\text {eff }}\right)$ & 2 \\
\hline $\begin{array}{l}\text { Perda da fibra nos comprimentos de } \\
\text { onda de bombeio }\left(\alpha_{p}\right)\end{array}$ & $0,3 \mathrm{db} / \mathrm{km}$ \\
\hline Coeficiente de espalhamento $\left(S_{c}\right)$ & 0,0022 \\
\hline
\end{tabular}

\begin{tabular}{|l|l|}
\multicolumn{2}{|c|}{ TABELA II. PARÂMETROS DA REDE ÓPTICA. } \\
\hline Parâmetros & Valores \\
\hline \multirow{2}{*}{ Comprimentos de Onda $($ em $\mathrm{nm})$} & $1546,99 \sim 1552,60 \mathrm{com}$ \\
& $\begin{array}{l}0,8 \mathrm{~nm} \text { de espaçamento entre } \\
\text { canais }\end{array}$ \\
\hline
\end{tabular}

\section{2) Parâmetros da rede de satélites $L E O$}

Os parâmetros de precipitação nas localidades que são nós com conexão com satélite estão na Tabela III.

TABELA III. PARÂMETROS DE PRECIPITAÇÃO.

\begin{tabular}{|l|c|c|}
\hline \multicolumn{1}{|c|}{ Cidade } & $\begin{array}{c}\text { Taxa de Precipitação } \\
(\mathrm{mm} / \mathrm{h})\end{array}$ & $\begin{array}{c}\text { Probabilidade de } \\
\text { Ocorrência }(\%)\end{array}$ \\
\hline Belém & 18 & 10 \\
\hline Recife & 22 & 10 \\
\hline Brasília & 10 & 30 \\
\hline Rio de Janeiro & 18 & 20 \\
\hline Curitiba & 16 & 20 \\
\hline Campo Grande & 12 & 10 \\
\hline Rondonópolis & 10 & 10 \\
\hline Cuiabá & 10 & 10 \\
\hline Porto Velho & 19 & 20 \\
\hline Rio Branco & 19 & 30 \\
\hline Santarém & 20 & 30 \\
\hline Manaus & 22 & 30 \\
\hline Macapá & 18 & 20 \\
\hline Boa Vista & 22 & 20 \\
\hline
\end{tabular}

Utilizamos valores típicos de parâmetros físicos para conexões em $30 \mathrm{GHz}$ (uplink) e $20 \mathrm{GHz}$ (downlink) [9]. Em enlace uplink, $G_{T}=76 \mathrm{~dB} ; P_{T}=100 \mathrm{~W} ; G_{s}=53 \mathrm{~dB} ; T_{s}=1000 \mathrm{~K}$; $B_{S}=350 \mathrm{MHz}$. Em enlace downlink, $G_{s}=49 \mathrm{~dB} ; P_{s}=6,3 \mathrm{~W}$; $G_{d}=72 \mathrm{~dB} ; T_{d}=250 \mathrm{~K} ; B_{r}=350 \mathrm{MHz}$. Para a largura de banda de $350 \mathrm{MHz}$, foi considerado o uso da taxa de 155 Mbps.

\section{A. Tráfego e Probabilidade de Bloqueio}

A distribuição de tráfego é heterogênea e proporcional ao número de habitantes apresentado na Tabela IV. A probabilidade, $P$, de um nó ser escolhido como gerador de tráfego ou como nó destino de uma determinada conexão é dado por

$$
P=\frac{H}{\sum_{n=1}^{N} H_{n}},
$$

na qual $H$ é o número de habitantes da cidade e e $H_{n}$ é o número de habitantes das $N=53$ regiões das cidades consideradas. 


\section{ALGORITMO}

Para cada enlace uplink ou downlink o seguinte procedimento é executado:

1. O intervalo de duração do enlace é dividido em 10 subintervalos de mesma duração. Os parâmetros físicos são verificados em cada subintervalo;

2. Para cada localização de estação terrestre é associada uma probabilidade de ocorrência de chuva e respectiva taxa de precipitação. No intervalo de duração do enlace a ocorrência ou não de chuva é verificada;

3. A relação $\mathrm{C} / \mathrm{N}$ é calculada em cada subintervalo e comparada com limiar;

4. A conexão é bloqueada se $\mathrm{C} / \mathrm{N}$ estiver abaixo do limiar $(50 \mathrm{~dB})$.

A Figura 3 mostra o fluxograma da verificação das restrições físicas das duas redes.

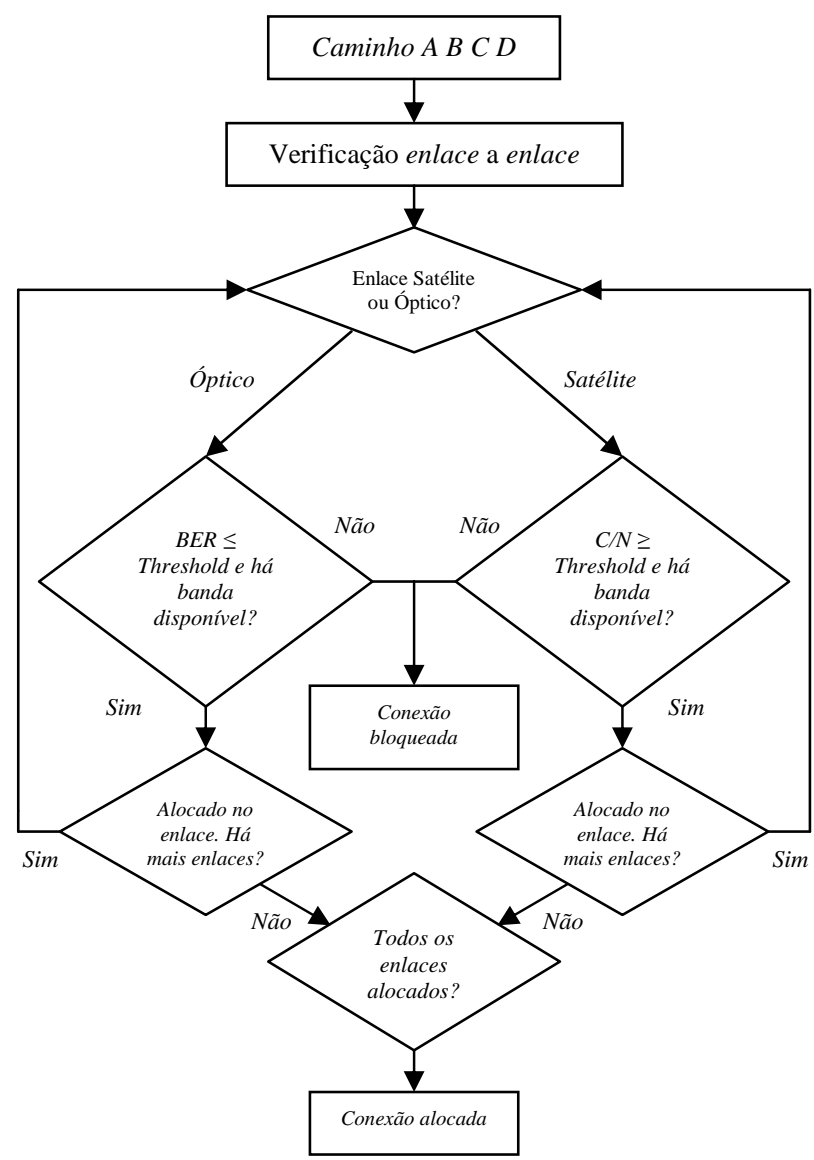

Figura 3. Fluxograma de verificações de restrições físicas.

\section{RESULTADOS}

Foram analisados separadamente o bloqueio de solicitações de conexão nas redes óptica e via satélite. O bloqueio na rede de satélite envolve as conexões que se originam em um nó que possui somente conexão com a rede de satélite e tem como destino nó óptico (ou vice-versa) e conexões que se originam de um nó que possui somente conexão com a rede de satélite e tem como destino um nó do mesmo tipo.

A rede via satélites passa a ser a rede auxiliar para as conexões que tem como nó fonte e nó destino somente nós ópticos. A rede auxiliar é utilizada pelo algoritmo, no caso de taxa de $155 \mathrm{Mbps}$, quando há indisponibilidade de recursos por um caminho totalmente óptico e disponibilidade de recursos na rede de satélites. A probabilidade de bloqueio global se refere todos os tipos de bloqueio. A análise de bloqueio de cada tipo de tráfego não é realizada neste trabalho devido ao espaço.

A Figura 4 mostra que a rede por satélites apresenta a menor probabilidade de bloqueio. Os nós fontes que possuem apenas conexão com satélite geram apenas tráfego com largura de faixa de $155 \mathrm{Mbps}$. A rede auxiliar apresenta os maiores valores porque como as conexões geradas pelos nós apenas com roteadores ópticos variam de $155 \mathrm{Mbps}$ até 2,5 Gbps, uma parte das chamadas geradas não pode ser atendida pela rede de satélites, cuja largura de faixa máxima é 155 Mbps por canal.

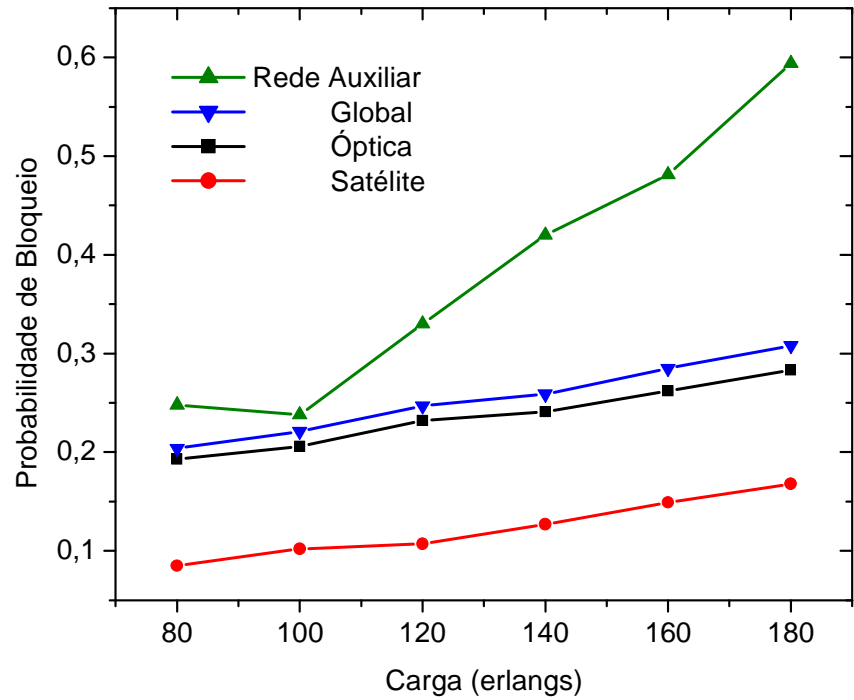

Figura 4. Probabilidade de bloqueio da rede.

Como pode ser observada pela Figura 5, a probabilidade de bloqueio por BER foi analisada para valores crescentes do $\mathrm{X}_{s w}$, que é o ruído crosstalk da chave do comutador OXC. Com o aumento do ruído, há o aumento da probabilidade de bloqueio por BER sendo que, a partir de $-15 \mathrm{~dB}$ o ruído $\mathrm{X}_{s w}$ torna-se um fator crítico para o funcionamento da rede óptica.

A Figura 6 mostra a probabilidade de bloqueio de conexões por BER para a configuração de conversão eletroóptica e agregação de tráfego. Observa-se bloqueio relativamente constante em torno de $4 \%$ para cargas entre 80 e 180 erlangs. Este fenômeno é provocado pela utilização de enlaces via satélite que diferenciam as possibilidades de caminhos para conexões de 155 Mbps. Neste tipo de enlace o valor da BER computado para as conexões é zerado nos nós e, desta maneira, muitas conexões que seriam bloqueadas pelo limiar de BER em caso de caminhos totalmente ópticos são alocadas em enlace via satélite.

A Figura 7 mostra a influência do aumento das taxas de precipitação iniciais da Tabela III sobre a probabilidade de bloqueio das conexões entre as estações terrestres e satélites para carga de 100 erlangs. A probabilidade de bloqueio aumenta e passa a valor praticamente constante a partir de $10 \%$ de aumento da precipitação. A partir deste ponto a 
atenuação provocada pela chuva afeta o enlace de maneira mais significativa, pois valores maiores de taxas de precipitação não implicam em uma grande diferenciação nos valores da probabilidade de bloqueio. Este fenômeno é devido à perda de qualidade de sinal (relação C/I) provocada pelo aumento dos valores da taxa de precipitação.

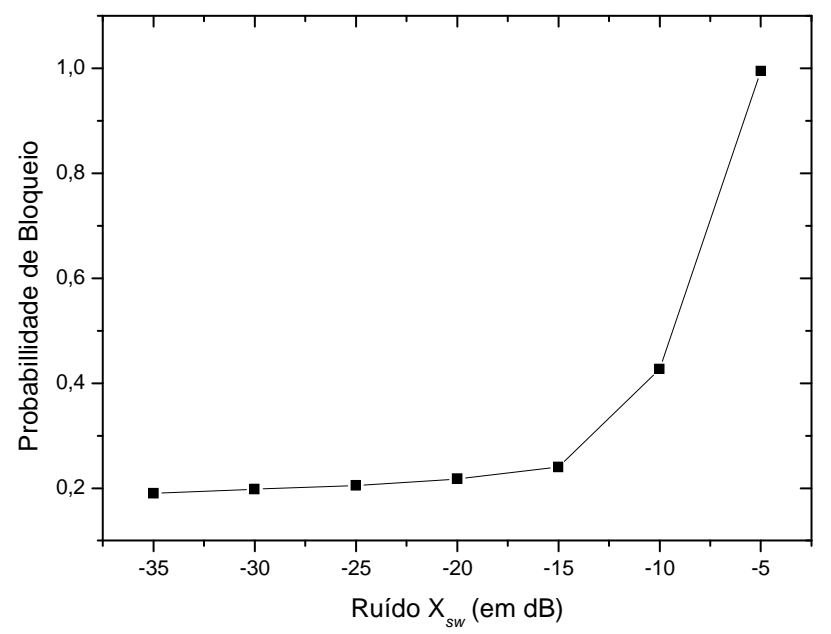

Figura 5. Probabilidade de Bloqueio por BER com aumento de $\mathrm{X}_{s w}$

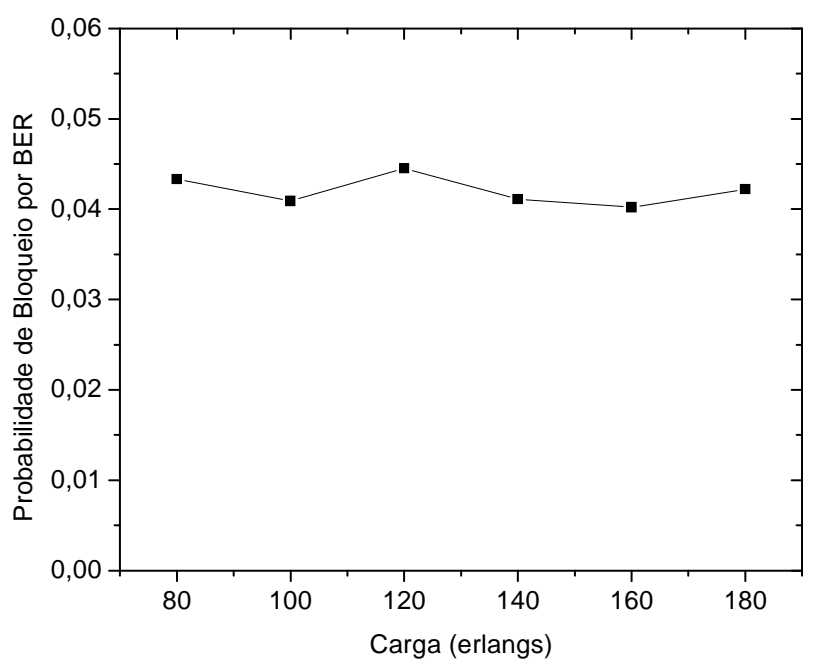

Figura 6. Probabilidade de bloqueio por BER.

O throughput da rede satélites foi analisado e seu comportamento em função do aumento da carga para os quatros canais dos satélites, totalizando $620 \mathrm{Mbps}$ para downlink e 620 Mbps para uplink, pode ser visto na Figura 8.

\section{CONCLUSÕES}

Neste artigo é apresentado o estudo da interligação de uma rede brasileira de comunicação óptica com uma rede de satélites do tipo LEO. A configuração da rede segue a apresentada por [7] e inclui restrições físicas inerentes às conexões com satélites.

A banda básica da rede óptica é 2,5 Gbps, com taxa total de 20 Gbps. Os nós da rede óptica (OXCs) são dotados de capacidade de realizar agregação de tráfego. As conexões com satélites LEO são atendidas por quatro canais de capacidade máxima individual $155 \mathrm{Mbps}$.

O desempenho da rede foi analisado para diferentes taxas de precipitação nas localidades onde se situam os nós com conexão com satélites. $\mathrm{O}$ aumento da probabilidade de bloqueio com o aumento das taxas de precipitação ocorre até valor em torno de $22 \%$, a partir do qual há estabilização por causa de pouca variação da relação $\mathrm{C} / \mathrm{N}$. Observa-se que os efeitos de atenuação por chuva afetam de maneira significativa o desempenho da rede óptica integrada com a rede de satélites.

A análise de bloqueio por BER para cada classe de tráfego estudada é a sugestão para continuação desta pesquisa.

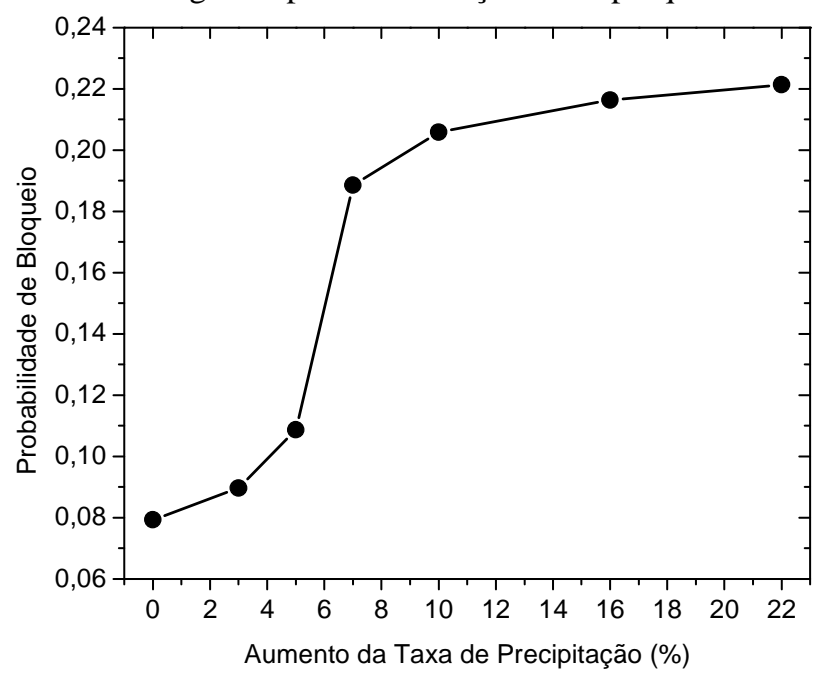

Figura 7. Probabilidade de bloqueio para aumento das taxas de precipitação. Carga 100 erlangs.

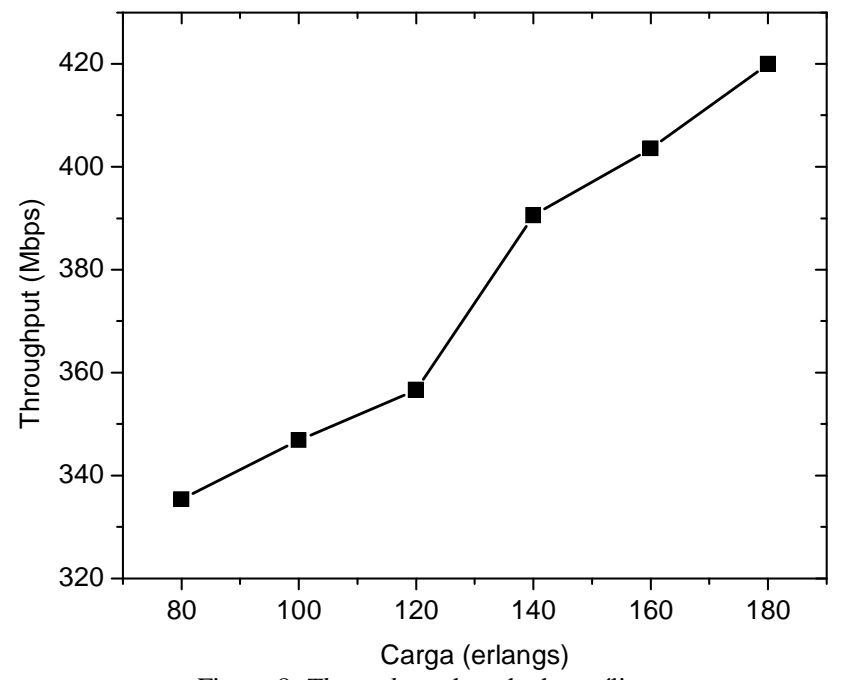

Figura 8. Throughput da rede de satélites.

\section{REFERÊNCIAS}

[1] H. Uzunalioglu, I.F. Akyildiz e M. D. Bender, "A routing algorithm for connection-oriented low earth orbit (LEO) satellite networks with dynamic connectivity", Wireless Networks, vol. 6, no. 3, pp. 181-190, 2000.

[2] M. O. Kolawole, "Satellite Communication Engineering", Marcel Dekker, Inc, 2002.

[3] Helvécio M. A. Neto, Eduardo M. G. de Queiroz, Amílcar C. César, "Análise de Custo-benefício de Rede Óptica WDM com Agregação de Tráfego e Agregação de Comprimentos de Onda”, XXV Simpósio 
Brasileiro de Telecomunicações-SBT'07, pp. 1-6 (CD-ROM), Recife, PE, 3 a 6 de setembro de 2007.

[4] Marcos A. C. Lima, Aluízio F.R. Araújo e Amílcar C. César, "Agregação Dinâmica de Tráfego em Redes Ópticas WDM sob Impacto de ASE e PMD utilizando Algoritmo Genético", XXI Simpósio Brasileiro de Telecomunicações-SBT'04, pp. 1-6 (CD-ROM), Belém, PA, 6 a 9 de setembro de 2004.

[5] D. Bisbal, I. Miguel, F. González, J. Blas, J.C. Aguado, P. Fernández, J. Durán, R. Durán, R.M. Lorenzo, E.J. Abril e M. López, "Dynamic routing and wavelength assignment in optical networks by means of genetic algorithms", Photonic Network Communications, vol. 7, no. 1, pp. 43-58, 2004.

[6] S. Nanba, S.Konish, S, Nomoto, "Optimum Traffic Distribution Algorithm for Multiple-Satellite Sytems Under Power Constraints",
International Conference on Communications, Vol. 22, no. 3, pp. 192500, 2004.

[7] Marcos A.C. Lima, Aluízio F.R. Araújo e Amílcar C. César, "Agregação Dinâmica de Tráfego em Rede Brasileira de Comunicação Óptica WDM e Via Satélite Utilizando Algoritmo Genético", XXII Simpósio Brasileiro de Telecomunicações - SBT'05, pp. 645-649, Campinas, SP, 4 a 8 de setembro de 2005 .

[8] Y. Huang, J.P. Heritage, B. Mukherjee, "Connection provisioning with transmission impairment consideration in optical WDM networks with high-speed channels", Journal of Lightwave Technology, vol. 23, no. 3, pp. 982-993, Março 2005.

[9] Randy H. Katz, "Mobile Satellite Systems", Class Lectures, University of California, 1996.

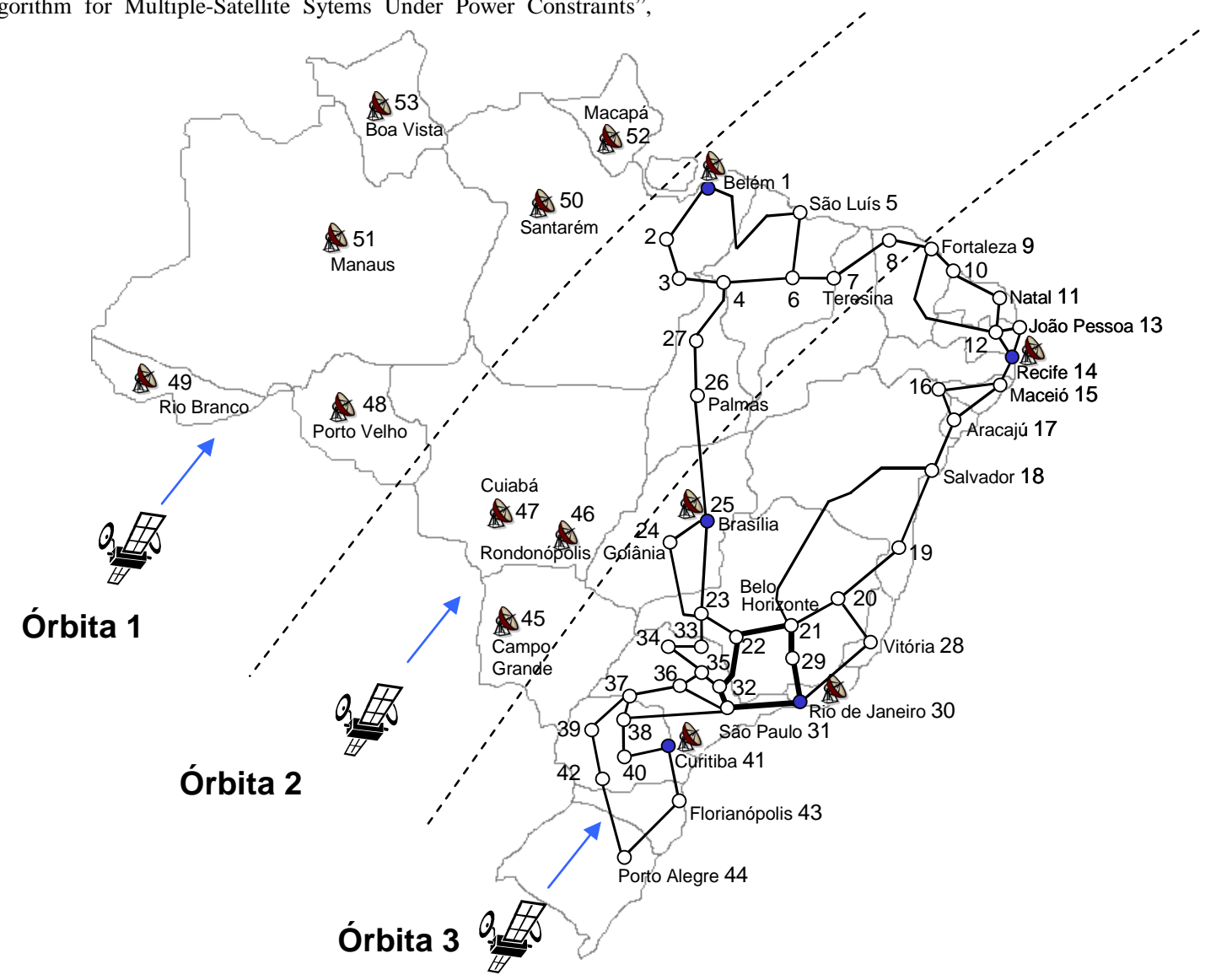

Figura 1. Rede híbrida brasileira formada por enlaces de fibra óptica e via satélite. Os nós roteadores em destaque são equipados com estaç̃os de comunicação por satélite. A rede óptica dispõe de 8 comprimentos de onda por enlace. Na rede via satélite há $\mathrm{C}=4$ transceptores disponíveis.

TABELA IV. NÚMERO DE MIL HABITANTES SERVIDOS PELOS NÓS ROTEADORES ${ }^{1}$

\begin{tabular}{|c|c|c|c|c|c|c|c|c|c|c|c|}
\hline Nó & Região & Habit. & Nó & Região & Habit. & Nó & Região & Habit. & Nó & Região & Habit. \\
\hline 1 & Belém & 4442,6 & 16 & Delm. Golveia & 144,3 & 31 & São Paulo & 30244 & 46 & Rondonópolis & 593,8 \\
\hline 2 & Tucuruí & 256,0 & 17 & Aracajú & 1784,4 & 32 & Campinas & 2809,8 & 47 & Cuiabá & 1910,5 \\
\hline 3 & Marabá & 582,8 & 18 & Salvador & 12577 & 33 & Rib. Preto & 1463,5 & 48 & Porto Velho & 1379,7 \\
\hline 4 & Porto Franco & 92,7 & 19 & Porto Seguro & 492,8 & 34 & São J. R. Preto & 1039,2 & 49 & Rio Branco & 557,5 \\
\hline 5 & São Luís & 4789,4 & 20 & Gov.Valadares & 1235,3 & 35 & São Carlos & 559,4 & 50 & Santarém & 910,8 \\
\hline 6 & Caxias & 769,3 & 21 & Belo Horizonte & 11189 & 36 & Bauru & 916,1 & 51 & Manaus & 2812,5 \\
\hline 7 & Teresina & 2843,2 & 22 & P. de Caldas & 677,9 & 37 & Cambará & 99,7 & 52 & Macapá & 477,0 \\
\hline 8 & Sobral & 502,3 & 23 & Uberlândia & 2505,3 & 38 & Ponta Grossa & 1199,4 & 53 & Boa Vista & 324,4 \\
\hline 9 & Fortaleza & 6928,2 & 24 & Goiânia & 5003,2 & 39 & Camp. Mourão & 352,7 & & & \\
\hline 10 & Mossoró & 641,1 & 25 & Brasília & 2051,1 & 40 & Guarapuava & 680,2 & & Reg. Sudeste & 72412 \\
\hline 11 & Natal & 2135,6 & 26 & Palmas & 634,4 & 41 & Curitiba & 6958,4 & & Reg. Norte & 12900 \\
\hline 12 & Campo Grande & 1283,6 & 27 & Araguaína & 522,6 & 42 & Pato Branco & 272,8 & & Reg. C. Oeste & 11636 \\
\hline 13 & João Pessoa & 2160,1 & 28 & Vitória & 3097,2 & 43 & Florianópolis & 5356,3 & & Região Sul & 25107 \\
\hline 14 & Recife & 7918,3 & 29 & Juiz de Fora & 2283,3 & 44 & Porto Alegre & 10187 & & Reg. Nordeste & 47741 \\
\hline 15 & Maceió & 2678,2 & 30 & Rio de Janeiro & 14391 & 45 & Campo Grande & 2078,0 & & Brasil & 169799 \\
\hline
\end{tabular}

Fonte: Censo IBGE 2000 